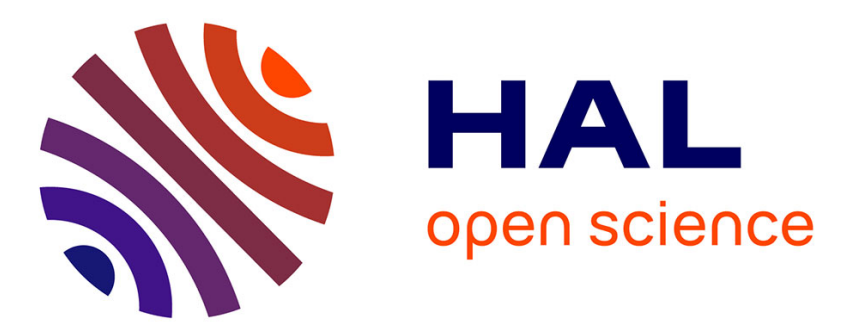

\title{
PENSER LE CORPS AFFECTIF COMME UN MEDIA
}

Fabienne Martin-Juchat

\section{To cite this version:}

Fabienne Martin-Juchat. PENSER LE CORPS AFFECTIF COMME UN MEDIA . CORPS : Revue Interdisciplinaire, 2008, Le Corps mangeant, 4, pp.85-92. 10.3917/corp.004.0085 . hal-01858670

\section{HAL Id: hal-01858670 https://hal.univ-grenoble-alpes.fr/hal-01858670}

Submitted on 22 Aug 2018

HAL is a multi-disciplinary open access archive for the deposit and dissemination of scientific research documents, whether they are published or not. The documents may come from teaching and research institutions in France or abroad, or from public or private research centers.
L'archive ouverte pluridisciplinaire HAL, est destinée au dépôt et à la diffusion de documents scientifiques de niveau recherche, publiés ou non, émanant des établissements d'enseignement et de recherche français ou étrangers, des laboratoires publics ou privés. 
Fabienne Martin-Juchat

Université Stendhal Grenoble 3

GRESEC - EA 608

Dans notre société occidentale, l'observation des pratiques de communication interpersonnelle, médiée ou médiatisée - nous montre que les affects du corps sont à la fois recherchés, survalorisés ou surexploités. L'objectif de cet article est de proposer un cadre théorique et méthodologique pour l'étude de ce phénomène ${ }^{1}$. Dans un premier temps, il s'agit d'observer la manière dont le corps et les affects ont été pensés en sciences humaines et sociales. Or, le corps et ses affects ont historiquement et majoritairement été traités séparément et, de surcroît, avec une épistémologie à dominante cognitiviste. À cet égard, les Sciences de l'Information et de la Communication (SIC) illustrent bien cette mouvance.

Dans le domaine de la communication interpersonnelle, le corps est entendu comme un système de codes à déchiffrer en vue d'une meilleure compréhension des messages verbaux. Dans le domaine de la communication de masse, seuls les discours verbaux et iconiques sont analysés dans leurs dimensions émotionnelles, mais en dehors de toute relation avec un corps qui éprouve ces discours dans les processus de réception. Corps, affects et communication ont été traités séparément, ce qui ne permet pas d'analyser les pratiques des affects du corps et donc d'en comprendre les finalités et leurs conséquences sociales.

Le qualificatif de non verbal (associé au para-verbal) est devenu le terme générique couramment employé pour désigner l'ensemble flou et global que forme le non-linguistique. Il y a le verbal qui est bien identifié et le non verbal, étudié en comparaison avec le verbal. Pourtant, comme le souligne M. Bernard, «l'apparence linguistique du jeu corporel autant que le silence de ses manifestations expressives sont le produit ou mieux l'artefact de l'impérialisme sémiotique du regard et surtout des discours idéologiques qui l'ont suscité et [...] qui l'ont conceptualisé sous des formes multiples et faussement contradictoires » (Bernard, 1985 : 360). De l'autre côté, les affects sont étudiés en communication de masse avec une forte influence de la psychanalyse et de la psychologie sociale qui réduisent très souvent la vie affective à des pulsions et des émotions primaires. Plus en avant, les affects ont une existence en dehors d'une chair éprouvée et donc d'un sujet construit par une culture et une histoire. "Il apparaît que la plupart des recherches traditionnelles sur l'affectivité, ses dimensions communicatives et interactives, sont négligées, et ceci pour deux raisons: l'orientation avant tout grammaticale des approches linguistiques, et la conception essentialiste de l'émotion qui est généralement à leur base, celle-ci étant perçue comme une expérience avant tout intrapsychique et individuelle. Par conséquent, les modèles traditionnels de l'affectivité ne peuvent saisir et décrire adéquatement la réalité communicative des émotions » (Drescher, 2003 : 169).

\section{LES SCIENCES DE LA CULTURE COMME PARADIGME}

Face à ce constat d'un manque théorique pour penser l'articulation entre corps, affects et communication, il importe de choisir un cadre épistémologique à partir duquel le corps et 
ses affects peuvent être pensés en co-imbrication dans les processus de communication. Le choix des sciences de la culture (Rastier, Bouquet, 2002) comme paradigme dominant, nous oriente vers la nécessité d'une compréhension des processus qui sont à l'origine de l'imbrication entre corps, affects et communication, afin de rompre avec le paradigme computationnel. Ce paradigme rencontre un certain succès. Que ce soit aux États-unis (avec F. Varela et surtout A. Damasio) ou en France (avec B. Andrieu, A. Berthoz, J. L. Petit pour la philosophie ; J. Fontanille et E. Landowski pour la sémiotique ; F. Rastier en sémantique ; J. Cosnier et B. Cyrulnik en éthologie de la communication), des chercheurs placent, avec des formulations qui leur sont propres, la question du corps en activité au cœur des processus d'interprétation et de communication sociale. Dans cette perspective des sciences de la culture, le cadre théorique choisi développe prioritairement des principes issus historiquement de la sociologie compréhensive et de la psychologie du développement en s'appuyant sur les recherches récentes en neurosciences. À partir de ce cadre théorique, nous pouvons redéfinir le rôle du corps et de ses affects dans les processus de cognition, de sémiotisation et de communication.

L'essentiel de l'ambition est de démontrer que la communication des affects et la communication par les affects constituent des processus anthropologiques. En conséquence, penser, au sein des sciences de la culture, le corps et ses affects dans cette perspective nous demande de croiser des théories qui le placent comme un objet à construire, tâche privilégiée des SIC $^{2}$. Dans une perspective anthropologique, il nous importera d'appréhender ce que font les acteurs du corps affectif en communication, leur «bricolage» (M. De Certeau), leur «aire avec » (J. Perriault).

\section{Aux origines du corps éprouvé}

Les conduites d'attachement sont des interactions affectives qui conditionnent l'apparition de la conscience à la fois réflexive puis projective, dont le langage. L'imitation joue un double rôle dans les différentes étapes du développement. Le penchant naturel à une imitation involontaire et immédiate manifeste un processus de fusion passionnelle qui engramme le corps de l'enfant. Puis, par les gestes imités et répétés, le nourrisson apprend à maîtriser les objets et à les transformer en symboles. L'imitation non consciente est passionnelle. La somme de mouvements empruntés à la chair empreinte, produit de l'imitation, constitue la naissance de la conscience en instaurant un différentiel dans la chair. Puis, l'imitation devient consciente et correspond à un mouvement de la chair dirigée par une in-tension que permet aussi l'émotion ${ }^{3}$. Ce processus affectivo-cognitif construit les représentations (selon B. Andrieu, A. Berthoz, J.L. Petit). Cette connaissance d'une coimbrication de l'affectif et du cognitif durant le développement du symbolique chez l'enfant nous permet de nourrir la thèse d'une chair mise en mouvement par des passions et des émotions non conscientes, ou corps éprouvé, dans tous les processus de cognition et de sémiotisation.

\section{Le Corps, médiateur affectif}

Le corps est éprouvé par des interactions affectives sécurisantes avec un corps construit socialement, la figure de l'attachement. En effet, les expressions affectives de la figure de l'attachement forment un récit culturel qui façonne la chair de l'enfant par un processus d'imitation non conscient. Ces empreintes corporelles, en tant que produit d'interactions affectives, vont permettre au nourrisson de développer ses capacités de perception puis de symbolisation, le construire socialement. Le corps éprouvé anthropologiquement devient un médiateur affectif, car il permet à l'individu de reconnaître 
ce qui fait sens pour lui, il est l'opérateur des relations entre valeurs abstraites, valeurs systémiques et valeurs d'usage selon L. F. de Saussure. Dans la relation, le corps exprime aussi différemment les types d'affects que sont les passions, les émotions et les sentiments (Martin-Juchat, 2005). Le corps est à la fois le médiateur à partir duquel l'individu peut conscientiser ses affects et le support de communication de ces derniers. L'éducation et la culture ont un rôle fondamental dans l'apprentissage de ces possibles accès. L'éducation se caractérise par l'incorporation d'habitudes sensibles ${ }^{4}$ qui correspondent à ce que l'individu doit ou ne doit pas percevoir comme informations affectives en provenance de son corps et de celui d'autrui. De la constitution (selon J.L. Petit) des informations en provenance du corps, sortes de cartes somatiques, se fait ensuite un processus de reconstruction mentale ou de génération de représentations, structuré par les langages et motivé par une tension affective.

Aussi, l'éducation par imitation corporelle, puis la société, par l'intermédiaire de ses pratiques, structurent la vie affective de l'enfant en structurant d'abord sa chair. L'adulte ne peut alors plus percevoir consciemment ${ }^{5}$ ce qui émerge d'une relation affective ou, inversement, peut savoir entrer volontairement dans ce type de relation, comme c'est le cas pour la transe ou le totémisme. Nous comprenons mieux les raisons du développement de techniques du corps (en référence à $M$. Mauss) visant à apprendre aux individus à être attentifs à leurs propres réactions, à intégrer les passions à un niveau sentimental de la conscience morale. Mais toute réintégration de la passion engendre des réactions affectives en boucle. En retenant l'envie d'action provoquée par la passion, l'individu peut ressentir une frustration, une culpabilité, du plaisir, ou les trois à la fois. Cependant, à partir du moment où il est conscient de la passion qu'il a ressentie, cela signifie qu'elle est devenue un sentiment de passion.

\section{QUELS USAGES DU CORPS AFFECTIF EN COMMUNICATION?}

La notion de corps affectif comme condensé de corps médiateur affectif est un exercice proche de ce que G. Granger (2002) appelle "l'imagination conceptuelle ». Ce condensé nous permet d'insister sur une imbrication ontogénétique : sans interaction affective, pas de corps perçu. Sans ce corps éprouvé, produit de relations historicisées, pas de processus de sémiotisation possible. D'un point de vue épistémologique, cela signifie que toute anthropologie trouve son origine fondatrice dans une intersubjectivité structurée par des corps affectifs en interaction. L'hypothèse n'est pas récente: G. Simmel puis G. Tarde en sociologie, T. Ribot, P. Janet et M. Jousse en psychologie du développement, M. Scheler et M. Merleau-Ponty en philosophie l'avaient déjà suggérée. Cela implique que les émotions sont des phénomènes sociaux avant d'être des états internes. Pourtant, domine toujours dans la recherche internationale une psychologisation des émotions dans la communication.

Dans une perspective propre aux SIC, la question des usages de ce corps affectif dans les processus de communication nous importe. Cette perspective peut se construire à partir de l'anthropologie du corps de D. Le Breton (1990), de l'anthropologie par la communication de Y. Winkin (1996), approches qui se situent toutes deux dans la continuité de l'interactionnisme symbolique. Nous allons donc explorer, dans la dernière partie de cet article, des exemples d'usages communicationnels du corps affectif. Aussi, c'est à partir d'une observation de ces usages que nous posons l'hypothèse centrale de cet article : les usages du corps dans différents dispositifs de communication nous montrent que le corps est exploité dans notre société occidentale comme un média caractérisé par un genre affectif de communication.

Les acteurs, par leur utilisation du corps affectif, le construisent comme un média. Cette affirmation repose sur une conception de média qui dépasse, bien entendu, celle d'être un simple support technologique, et s'appuie au contraire sur celle de J. Davallon (1999) et de 
B. Miège (1989) : un média est un dispositif symbolique qui développe des pratiques qui lui sont spécifiques par l'intermédiaire d'un genre qui est à définir. Aussi, nous employons dans cet article le terme de média dans une acception qui remet en cause la vision de média propre à une sociologie empirique d'héritage nord américain ${ }^{6}$. Cette vision, revisitée en France par les chercheurs en SIC, consiste à insister, au-delà des contraintes que font peser les médias, sur les mécanismes de la réception, sur la nécessité de penser la pratique médiatique comme une pratique symbolique sociopolitique. Il s'agit, dans les termes de B. Miège, d'appréhender toute la complexité du social en jeu dans les dispositifs de réception médiatique appréhendés comme des systèmes symboliques.

Il nous appartient maintenant pour clore cet article synthétique de présenter des exemples de situations où le corps est exploité comme le support privilégié de la transmission d'une argumentation persuasive, par l'intermédiaire de son genre affectif de communication qui lui est spécifique. Nous dissocierons les pratiques du corps affectif comme média : - en représentation (construit par les marques et les médias); - en interactions interpersonnelles (construit par les individus) ou immergé dans des dispositifs (construit par les industriels). Enfin, nous esquisserons des raisons sociétales qui justifient une tendance à la construction du corps affectif comme média, ou plus précisément : à la surexploitation et la survalorisation des affects du corps dans les représentations et les interactions, à la recherche d'un corps éprouvé comme finalité interactionnelle, en particulier lors des processus de réception.

\section{En communication de masse}

Les émissions télévisuelles autour du concept de télé-réalité médiatisent le mieux des normes de comportement en ayant recours au corps affectif. Ces constructions transmettent des normes selon les sexes, les genres, les sous-cultures, que les téléspectateurs vont intégrer, adapter, rejeter dans leurs pratiques de communication. Dans le domaine publicitaire, ce sont les marques qui exploitent le corps dans l'objectif de transmettre, de diffuser, voire de prescrire un système de valeurs idéologiques par l'intermédiaire de mises en scène de corps en interaction (Martin-Juchat, 2004). Ces pratiques sollicitent un corps-machine, un corps fragmenté car composé selon des règles, socioculturellement prédéfinies, de représentation sentimentale de la passion, de l'émotion et du sentiment. Ces discours participent à la perduration d'une vulgate sur un corps-objet, un corps-artefact, car reconstruit artificiellement. Cet artefact est devenu le support privilégié de la diffusion de normes par son genre affectif de communication. Mieux encore, les marques, par le média publicitaire, normalisent ou encore codifient non seulement les codes de représentation des affects du corps, mais aussi les critères de reconnaissance visuelle de ces derniers.

Aussi, la publicité sert aujourd'hui beaucoup moins à vendre qu'à diffuser des normes de comportement qui incarnent les valeurs identitaires des marques et des normes de reconnaissance visuelle de ces dernières. Les individus, et surtout les jeunes, regardent la publicité (ou d'autres récits fictionnels tels que les séries ou la télé-réalité) pour apprendre des modes de comportement relationnel à partir desquels ils pourront s'identifier par imitation. Nous retrouvons ici une des fonctions fondatrices de l'affectivité qui est de construire l'individu en tant qu'être social en mettant en mouvement sa chair. Comme le souligne D. Pasquier, « il ne fait pas de doute qu'une série comme Hélène identifie de manière particulière et spécifique certains problèmes (en l'occurrence comment ajuster le comportement féminin sans bouleverser les relations de couple) et propose des solutions à ces problèmes (notamment un repli sur des positions moins revendicatives et plus maternelles à l'égard des hommes). Il ne fait pas de doute non plus que ces propositions ont des conséquences sur la manière dont les petites correspondantes envisagent leur propre corps et leur propre vie » (Pasquier, 1999 : 222). 


\section{En communication interpersonnelle}

Le corps est devenu le support privilégié de l'expression et de la diffusion d'une identité sociale. Il s'agit de contrôler les expressions affectives du corps en tant que médium de compréhension et surtout de diffusion de cette identité. La quantité pléthorique de livres ${ }^{7}$, de dossiers de magazines, d'offres et de demandes en formation pour adulte, ou encore en coaching individuel montre à l'évidence que gérer l'émotion est un objectif collectif. Cette gestion de l'émotion est véritablement hypermoderne car elle se caractérise par un paradoxe (Lypovetsky, Charles, 2004). En effet, il s'agit pour l'individu occidental d'une part d'apprendre à gérer l'expression corporelle de ses affects, d'autre part de savoir se laisser aller à leur expression, se mettre à l'écoute de ses manifestations. Les problèmes méthodologiques quant à ce type d'étude sont nombreux. L'accès à la vie de l'entreprise n'est pas simple. Les études, terrain d'observation des pratiques relationnelles dans ce milieu, comme dans d'autres d'ailleurs, sont difficiles à mener. À cet égard, la télé-réalité est paradoxalement (car ce n'est pas sa finalité première) une source précieuse pour observer la manière dont les individus gèrent leurs affects dans différents types de situations. Cependant, ces interactions affectives constituent autant de pratiques culturelles qui manifestent le social en cours de construction. Ces corps mis en mouvement manifestent toute la complexité des phénomènes d'interprétation : communiquer, c'est être éprouvé corporellement par l'orchestre ${ }^{8}$.

\section{Au sein des dispositifs socioculturels}

Les magasins, les lieux d'expositions culturelles dont les musées, les centres de loisirs ou parcs d'attractions sont des espaces privilégiés où il est possible d'appréhender non seulement le corps en représentation mais aussi la manière dont le corps est éprouvé par l'acte de réception ${ }^{9}$. Il peut paraître choquant de mettre sur le même registre une enseigne comme Planet Saturn ou Sephora et un musée, et pourtant, du point de vue de leur mode de communication, même si les finalités diffèrent, ces espaces méritent d'être comparés. Les espaces scéniques commerciaux construits par des techniques de communication issues du marketing expérientiel, nommé aussi sensoriel, ou encore émo-marketing (Cobé, 2004 ; Rieunier, 2002 ; Hetzel, 2002 ; Cova et Cova, 2001), demandent de nous interroger sur le type de relation qu'ils induisent et construisent dans leurs usages, et sur les conséquences sociales de cette relation.

La question qu'il est alors possible de se poser est la suivante : en quoi les mises en scène communicationnelles d'un produit culturel et d'un produit commercial diffèrent-elles quant à leurs capacités à éprouver les corps dans la relation de réception ? Afin de répondre à cette question, il s'agira de construire des études de terrain visant à évaluer les enjeux de la rencontre des univers des marques, des médias et de la culture, à mieux préciser le périmètre de leurs champs d'action, leurs modes et leurs limites et enfin de mesurer, quant à leur réception, la manière dont ils éprouvent les corps afin de transmettre des savoirs. À cet égard, nous soutenons l'idée, en nous appuyant sur une critique de la vision française nihiliste, ou nostalgique, de la postmodernité ou de l'hypermodernité ${ }^{10}$, que les pratiques d'exposition définies a priori comme commerciales génèrent aussi du sens partagé et de la relation sociale. Ces pratiques sont en voie de devenir les nouveaux lieux de diffusion, de vulgarisation et de partage des savoirs et « la corporéité sera l'ambiance générale dans laquelle corps individuels, sociaux et mystiques se situent les uns par rapport aux autres. Et ce sera l'horizon de communication qui servira de toile de fond à l'exacerbation des apparences » (Bayard, 1992 : 28).

\section{L'AFFIRMATION D'UNE IDEOLOGIE SOCIETALE}


L'hypermodernité selon G. Lipovetsky se définit par une exacerbation des valeurs de la postmodernité et l'apparition de paradoxes engendrés par leur mise en pratique. À cette définition, nous pouvons ajouter que le corps, par son genre affectif de communication, est devenu, dans cette société, à la fois la surface, la trame, l'objet, le dispositif qui incarne, cristallise ou encore met en scène les figures et les normes d'une époque mais aussi la manière dont les individus les incorporent. En guise d'ouverture, à partir de ces quelques exemples d'usages communicationnels du corps affectif, nous pouvons appréhender les raisons sociétales de sa construction comme média.

- Les marques, les médias éprouvent les corps dans la pratique médiatique en ayant recours à des corps en représentation, car le corps éprouvé est le médiateur des connaissances. Dans cette logique, il s'agit d'éprouver les corps pour marquer les esprits.

- Les marques et les médias exploitent le corps affectif comme support d'une argumentation idéologique car, dans notre société, son interprétation (par identification et projection affectives) génère plus de valeur de vérité et de confiance que tout autre genre de discours.

- Les individus valorisent les caractéristiques du corps affectif car ce dernier leur permet de mettre en scène (au sens de E. Goffman) leurs aspirations idéologiques.

Aussi, que le corps soit devenu le média privilégié de la production d'un discours social ou d'un mode de propagande est une conséquence évidente. Le recours au corps est devenu monnaie courante lorsque les acteurs ont besoin d'incarner une idéologie. Le corps, parce qu'il transmet du sens par fusion, projection ou identification affectives, est un puissant outil de transmission d'une idéologie. Enfin, ce phénomène est accentué car, dans notre société hypermoderne, les expressions affectives du corps ont plus valeur de vérité que les expressions verbales ${ }^{11}$. Le discours corporel est interprété comme un acte sincère, moins soumis à la manipulation. Cela renvoie à une conception d'un corps qui ne peut pas mentir, qui dit toujours la vérité, idéologie caractéristique de ce début de $\mathrm{XXI}^{\mathrm{e}}$ siècle.

\footnotetext{
${ }^{1}$ Cet exposé est un résumé retravaillé d'un mémoire d'Habilitation à Diriger des Recherches (Martin-Juchat, 2005).

${ }^{2}$ Sciences de l'information et de la communication.

${ }^{3}$ Dans la continuité des écrits d'A. Damasio (2002), il convient de distinguer : un état d'émotion qui peut être déclenché et exécuté de façon non consciente ; un état de sentiment de l'émotion qui peut être représenté de façon non consciente ; un état de sentiment rendu conscient dont l'organisme, qui a simultanément émotion et sentiment, prend conscience.

${ }^{4}$ Selon P. Bourdieu, cet hexis corporel, ou ensemble de conduites fortement intériorisées, engrave la condition sociale, et constitue les raisons fondatrices des logiques de reproduction des inégalités. Or, comme nous l'observerons ultérieurement, les usages du corps affectif manifestent aussi une négociation en cours de cet ordre social. Dans des termes simmeliens, nous dirions que les jeux affectifs corporels sont des lieux où se manifeste la négociation de l'ordre social.

${ }^{5}$ «L'homme normal est joué par le Réel qui se réverbère en lui. Le Réel est en lui, sans lui, malgré lui. Cela se sait en lui, mais il n'est pas toujours en éveil à cette prise de conscience» (Jousse, $1969: 56$ ).

${ }^{6}$ Cette sociologie positiviste et critique appréhende l'efficacité médiatique en termes de manipulation d'une masse passive (Quéré, 1982).

${ }^{7}$ Dont le plus médiatisé est le livre de D. Goleman qui date de 1997.

${ }^{8}$ Pour reprendre l'expression de Y. Winkin tout en réintégrant la thèse de G. Simmel.

${ }^{9}$ Dans la continuité des travaux de S. Chaumier (2005).

${ }^{10}$ Critique des visions ethnocentrées présentes chez D. Le Breton, M. Maffesoli et surtout J. Baudrillard, développée par Kroker, A., Cook, D, 1986, The postmodern Scene, New World Perspectives, Montréal - Turner, B., 1987, « A note on Nostalgia », Theory/Culture/Society, vol. 4, pp. 147-156, résumée par C. Bayard (1992).
} 


\footnotetext{
${ }^{11}$ À propos de la télé-réalité, «le corps semble bien du coup servir de sésame par lequel on accède « vraiment » tout en mettant entre parenthèses les identités sociales » dans Duret, Roussel, $2003: 18$.
}

\section{BIBLIOGRAPHIE}

Andrieu B. 2002, La chair du cerveau : philosophie et biologie de la cognition, Liège, Sils Maria.

Andrieu B. 2003, Le laboratoire du cerveau psychologique: histoire et modèles, Paris, CNRS.

Bayard C. 1992, «Epistémologie du corps et postmodernité : de l'apocalypse de Baudrillard à l'Einfühlung de Maffesoli » dans Sociologies et sociétés, vol. XXIV n¹ : pp. 19-32.

Bernard M. 1985, L'expressivité du corps : recherche sur les fondements de la théâtralité, Paris, Chiron.

Berthoz A. 1997, Le sens du mouvement, Paris, Odile Jacob.

Berthoz A. et Jorland G. (éds.), 2004, L'empathie, Paris, Odile Jacob.

Chaumier S. (dir.), 2005, Du musée au parc d'attractions. Culture et Musées, n ${ }^{5}$, Actes Sud.

Cobé M. 2004, Emotional Branding: the New Paradigm for Connecting Brands to People, Hardcover.

Cosnier J. 1998, Le retour de psyché, Paris, Desclée de Brouwer.

Cova B. et Cova V. 2001, Alternatives Marketing, Paris, Dunod.

Cyrulnik B. 1997, L'ensorcellement du monde, Paris, Odile Jacob.

Damasio A. R. 2001, L'erreur de Descartes : la raison des émotions, Paris, Odile Jacob (1 $1^{\text {ère }}$ éd. 1995).

Damasio A. R. 2002, Le sentiment même de soi : corps, émotions, conscience, Paris, Odile Jacob (1 ${ }^{\text {ère }}$ éd. 1999).

Damasio, A. R. 2003, Spinoza avait raison : joie et tristesse, le cerveau des émotions, Paris, Odile Jacob.

Davallon J. 1999, L'exposition à l'oeuvre: stratégies de communication et médiation symbolique, Paris, L'Harmattan.

Drescher M. 2003, «La dimension interactive de l'investissement affectif » dans J. M. Colletta et A. Tcherkassof (éds.), Les émotions : cognition, langage et développement, Bruxelles, Mardaga, pp. 166-173.

Duret P. et Roussel P. 2003, Le corps et ses sociologies, Paris, Nathan Université, coll. 128.

Fontanille J. 2004, Soma et Séma, Paris, Maisonneuve \& Larose.

Goleman D. 1997, Emotional Intelligence: why it can matter more than IQ, New York, Bantam Books.

Granger G. 2002, Sciences et réalité, Paris, Odile Jacob.

Hetzel P. 2002, Planet Conso : marketing expérientiel et nouveaux univers de consommation, Paris, Éditions d'Organisation.

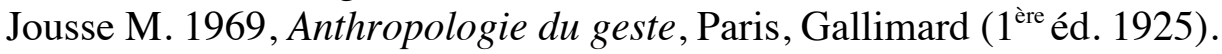

Landowski E. 2004, Passions sans nom, Paris, PUF.

Le Breton D. 1990, Anthropologie du corps et modernité, Paris, PUF.

Lypovetsky G. et Charles S. 2004, Les temps hypermodernes, Paris, Grasset.

Martin-Juchat F. 2004, «Sexe, genre et couple en publicité : une tendance à la confusion » dans Sexe et Communication, revue MEI n²0, Froissard P. et Bouchard J. (éds.) : pp. 61-82. 
Martin-Juchat F. 2005, Penser le corps affectif comme média, HDR en SIC de l'Université de Bourgogne.

Martin-Juchat F. 2005, «Représentations visuelles des corps en interaction : quels modèles de l'interprétation?» publié par l'International Society of Interacting Bodies : http://gesturelyon2005.ens-lsh.fr/article.php3?id_article $=259$.

Pasquier D. 1999, La culture des sentiments : l'expérience télévisuelle des adolescents, Paris, MSH.

Miège B. 1989, La société conquise par la communication, Grenoble, PUG.

Quéré L. 1982, Des miroirs équivoques : aux origines de la communication moderne, Paris, Aubier.

Rastier F.et Bouquet S. (éds.) 2002, Une introduction aux sciences de la culture, Paris, PUF. Rieunier S. 2002, Le marketing polysensoriel du point de vente : créer et gérer l'ambiance des lieux commerciaux, Paris, Dunod.

Petit J. L. 2004, «Empathie et intersubjectivité » dans Berthoz A. et Jorland G. (éds.), L'empathie, Paris, Odile Jacob : pp. 123-148.

Winkin Y. 2001, Anthropologie de la communication. De la théorie au terrain, Paris, Seuil (1996 $1^{\text {ère }}$ éd.). 